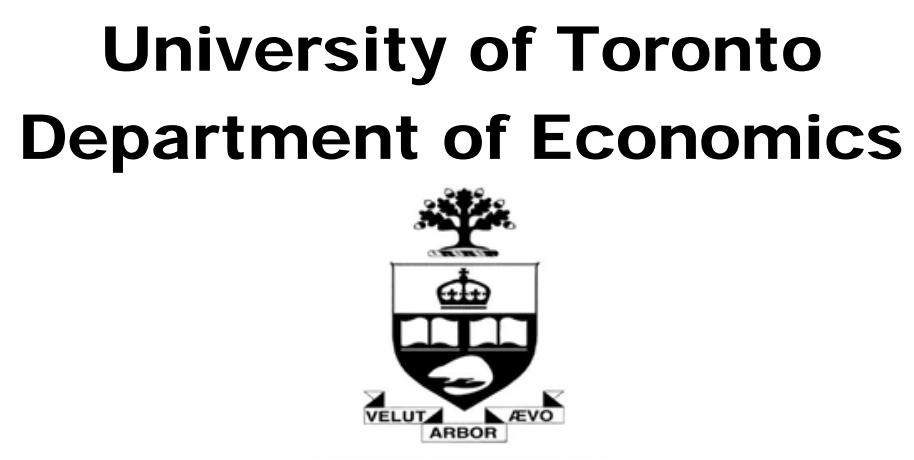

Working Paper 296

Party formation in single-issue politics [revised]

By Martin J. Osborne and Rabee Tourky

July 13,2007 


\title{
Party formation in single-issue politics
}

\author{
MARTIN J. OSBORNE \\ Department of Economics, University of Toronto \\ RABEE TOURKY \\ Department of Economics, University of Queensland
}

July 12, 2007

\begin{abstract}
We study the implications of economies of party size in a model of party formation. We show that when the policy space is one-dimensional, candidates form at most two parties. This result does not depend on the magnitude of the economies of party size or sensitively on the nature of the individuals' preferences. It does depend on our assumptions that the policy space is one-dimensional and that uncertainty is absent; we study how modifications of these assumptions affect our conclusions.
\end{abstract}

KEYWORDS. Political parties.

JEL CLASSIFICATION. D70, D72.

\section{INTRODUCTION}

In many societies collective decisions are made in legislative assemblies, typically by voting among the legislators. In most such assemblies, legislators are

Martin J. Osborne: martin. osborne@utoronto.ca

Rabee Tourky: r.tourky@uq. edu . au

An early version of this paper, circulated in February 2002, was entitled "Party formation in collective decision-making". Osborne started this research during a visit to the University of Melbourne, and continued it during a visit to the Australian National University. He thanks both institutions for their generous hospitality. He also gratefully acknowledges financial support from the Connaught Foundation and the Social Sciences and Humanities Research Council of Canada. Tourky is grateful for financial support from the Australian Research Council. We are grateful to Wing Suen and two referees for helpful comments and for pointing out errors in a previous version.

Copyright (c) 2007 Martin J. Osborne and Rabee Tourky. 
grouped into parties. Why do parties exist and what determines their number and positions?

We explore an economic rationale for parties. ${ }^{1}$ To get elected to a legislature, candidates must inform citizens of their platforms, persuade citizens to support them, and get their supporters to the polls. These activities are all costly, and although some of the cost may be offset by public subsidies, a candidate typically bears a significant burden herself. We study the implications of the cost borne by a candidate decreasing in the number of candidates fielded by the party to which the candidate belongs. The main reason we have in mind for such "economies of party size" is that fixed costs are shared within a party. National advertising, for example, is largely independent of the number of candidates in a party. In addition, if contributors to a party are motivated partly by the possibility of the party's gaining power and passing legislation favorable to them, and large parties are disproportionately powerful, then large parties may attract more contributions per candidate than small parties, also effectively creating economies of party size. All of our results depend only on the existence of economies of party size, not on their magnitude.

Our model, an extensive game with two stages, is illustrated in Figure 1. In each stage, actions are taken simultaneously. In the first stage each member of a set of politicians chooses whether to stand for election, and if so which position to champion, where championing a position $x$ entails committing to vote, if elected to the legislature, according to single-peaked preferences centered at $x$. In the second stage each member of a set of citizens chooses whether to vote, and if so for which candidate. A deterministic rule translates votes into a set of elected candidates, whom we call "legislators". We assume that the policy outcome is the median of the positions championed by the legislators. All politicians and citizens care only about the policy outcome-no one is under any illusion about the implications of their actions. (In particular, no citizen votes for a candidate merely because the candidate's announced position is similar to the citizen's favorite position; rather, each citizen considers the implication of her vote for the final legislative outcome.) We impose no assumptions on the politicians' and citizens' preferences beyond specifying their domain. Thus all our results hold for any preferences.

\footnotetext{
${ }^{1}$ Several other reasons for parties have been studied; we discuss the literature in Section 2.
} 


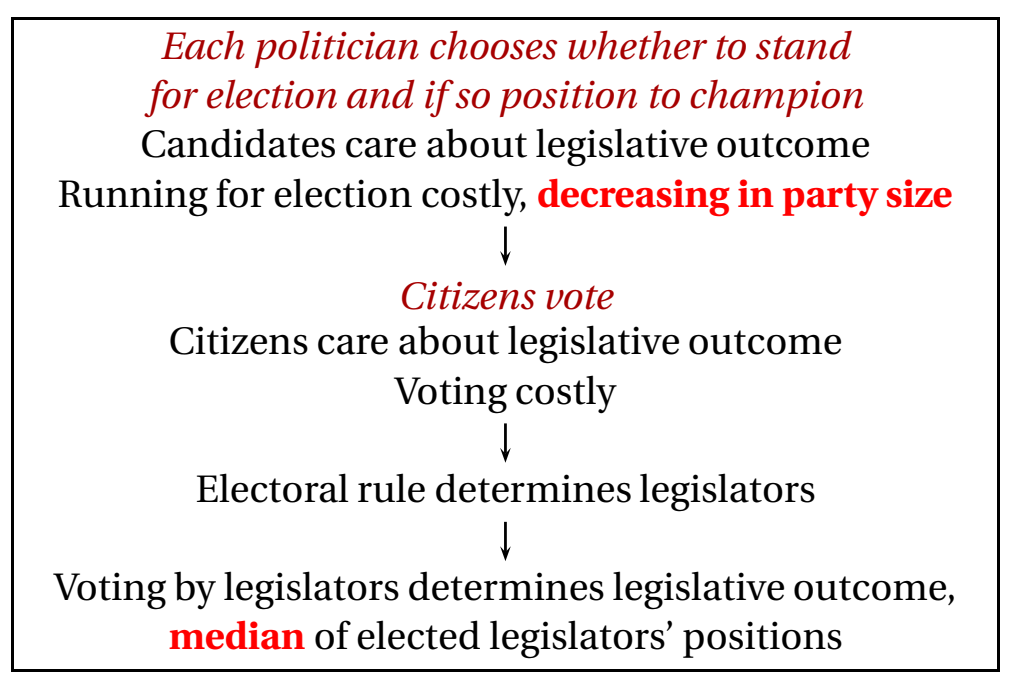

FIGURE 1. An outline of the model. The strategic choices are in italics and two key features of the model are in boldface.

We assume that the policy outcome is the median of the positions championed by the legislators because we have in mind that this outcome is determined by a majority vote among the legislators, or a sequence of such votes. We do not model the voting procedure explicitly, but rely on existing results to justify our assumption. (Among an odd number of legislators, the median position beats every other under majority rule pairwise voting. More generally, the median is the only subgame perfect equilibrium outcome of any "binary agenda" (a procedure in which the outcome is the result of a sequence of pairwise votes) in which the players use weakly undominated strategies (see, for example, Miller 1995, Section 6.3).)

We define a party to be a group of legislators who are committed to vote according to single-peaked preferences centered at the same position. In an equilibrium, no politician can deviate to champion a different position and induce a better outcome according to her preferences, given the other politicians' positions. The strategic options of party members in the world are richer-for example, they include coordinated changes in the members' positions. Parties are also long-lived institutions, whereas our model is static. We comment on these points in Section 8. 
The rule that determines the candidates who are elected, given the number of votes received by each candidate, is designed to capture simply the idea that the elected candidates are the ones who get the most votes. It is formulated abstractly; it is intended not to model a specific voting rule, but rather to capture the main features of a range of electoral systems. The rule is that a candidate is elected if and only if she obtains at least some quota of votes. We assume that this quota is a continuous nondecreasing function of the profile of vote totals. An example is the function that assigns to each vote profile the maximal number of votes obtained by any candidate in that profile. In this case a candidate has to obtain at least as many votes as any other candidate to get elected-she has to be "first past the post"; in the event of a tie, many candidates may satisfy this criterion. Another example is the function that assigns to each vote profile the total number of votes divided by a fixed number, which defines a voting rule that models a simple form of "proportional representation".

The solution concept we apply to the game is a variant of Nash equilibrium. The voting subgames, following the candidates' choices of policies to champion, may have multiple Nash equilibria, causing the whole game to have many Nash (and even subgame perfect) equilibria. In particular, the game may have equilibria in which a potentially profitable deviation by a candidate is deterred by a change in the citizens' voting behavior even though the original voting behavior is an equilibrium of the subgame to which the deviation leads and, if the citizens adhere to this behavior after the deviation, the policy outcome remains the same. We exclude such equilibria by assuming that the citizens do not change their voting behavior following a deviation by a candidate unless either a change is necessary because the original behavior is no longer a Nash equilibrium or, even though the original behavior remains an equilibrium, the policy outcome changes.

To describe our solution concept more precisely, suppose that a move by candidate $i$ changes the profile of the candidates' actions from $a$ to $a^{\prime}$. Denote by $b$ the citizens' voting profile following $a$. Suppose that $i$ 's move does not affect the policy outcome if the same candidates continue to be elected and that $b$ is an equilibrium of the subgame following $a^{\prime}$. Then we insist that the citizens' voting profile in the subgame following $a^{\prime}$ is $b$. We call Nash equilibria with this property subgame persistent equilibria. 
Our main result (Proposition 1) is that if the policy space is one-dimensional (i.e. there is a single political issue) then any subgame persistent equilibrium involves at most two parties, and if there are two parties then these parties have approximately the same number of candidates. In addition, some candidates may run as independents; if there are two parties, then there are at most three independents, whose positions lie between those of the parties. If the cost of running as an independent is high enough, then all subgame persistent equilibria involve two equal-sized parties. These results hold no matter how small the economies of party size. The positions of the candidates in an equilibrium depend on the magnitude of these economies, but the qualitative characteristics of the equilibria do not.

The main idea behind this result is simple. Suppose that the positions of two parties are to the left of the median position of all elected candidates. Suppose that a candidate from the smaller of these parties moves to the other party. If the set of elected candidates (including the one who moved) remains the same after the candidate moves, then the outcome chosen by the legislature (the median of the elected candidates' positions) also remains the same. Hence, given that the party to which the candidate moves is at least as large as her original party and given economies of party size, the candidate is better off, regardless of her preferences: her move does not affect the outcome, and her cost of running decreases.

To use this line of argument to delimit the set of equilibria, we need to consider the circumstances under which a move by a candidate has no effect on the set of elected candidates. Under subgame persistence, the set of elected candidates remains the same after a candidate's move if the citizens' voting behavior remains an equilibrium and, if the citizens adhere to this behavior, the policy outcome remains the same. Thus we need to consider how a candidate's move affects the citizens' incentives to vote for each candidate.

To illustrate these incentives, suppose there are eight candidates, two at each of four distinct positions. Suppose that in the equilibrium of the voting subgame, all candidates obtain the same number of votes and are elected. ${ }^{2}$ Suppose that

\footnotetext{
${ }^{2}$ For some specifications of the citizens' preferences and quota function, the voting subgame has such an equilibrium. In any equilibrium, the elected candidates tie. This feature of an equi-
} 
one of the candidates at the leftmost position, say candidate $i$, moves to the center-left position. We claim that this move has no effect on the citizens' voting incentives. Specifically, for any deviation $d$ by a small group of citizens after $i$ moves there is a deviation $d^{\prime}$ (which may be equal to $d$ ) by the same group before $i$ moves that induces the same change in the outcome. If, for example, a small group of citizens voting for one of the four leftmost candidates switches to abstention and this switch causes the candidate for whom they were voting to no longer be elected, then the median changes to the position of the center-right candidates both before and after $i$ moves. Given that $i$ 's move does not affect the citizens' voting incentives, their original voting behavior remains an equilibrium when $i$ moves, so that in a subgame persistent equilibrium $i$ remains elected after her move; because she becomes a member of a larger party, she is better off, so that the original configuration of the candidates' positions is not consistent with equilibrium.

Now suppose there are three candidates, who take distinct positions, and that in the equilibrium of the voting subgame all three obtain the same number of votes and are elected. If the left candidate, say candidate $i$, moves to the position of the middle candidate, she would be better off if all three candidates were to remain elected. But in this case, the citizens' incentives to vote do not remain the same, so that we cannot conclude that all three candidates remain elected. Specifically, a switch to abstention by citizens voting for the right candidate has different effects before and after $i$ 's move. It causes the candidate on the right not to be elected, ${ }^{3}$ which before $i$ 's move changes the policy outcome from the position of the middle candidate to the average of the positions of the left and middle candidates, but after $i$ 's move has no effect on the outcome. Thus after $i$ 's move, the original voting equilibrium is no longer an equilibrium, and hence the citizens' voting behavior must change. In the new equilibrium, $i$ may not be elected, in which case her move would be undesirable. Hence we cannot rule out the original configuration of the candidates' positions as an equilibrium.

librium is a consequence of our assumption of perfect information. In the presence of uncertainty, exact ties would no longer be a feature of the equilibria.

${ }^{3}$ Note that the citizens' switch to abstention must affect the outcome, otherwise it would be profitable (because voting is costly), contradicting the fact that the voting profile is an equilibrium. 
It turns out that many moves by candidates, like the one in the first example, do not affect the outcomes citizens can induce by changing their voting behavior, so that we are able to significantly restrict the set of equilibrium configurations of the candidates' positions, in particular ruling out all configurations in which there are more than two parties.

We have assumed so far that candidates can commit to the positions they choose. One way to deal with the case in which they cannot commit to these positions is to require that after an election, no candidate can, by changing her position, induce an outcome that she prefers, given the other candidates' positions. If the candidates' positions satisfy this condition, we say that they are "incentive compatible". Because we impose no assumptions on the candidates' payoff functions, the condition does not directly restrict the qualitative characteristics of the equilibria. However, in an environment in which candidates cannot commit to positions, we need to modify our solution concept to reflect the fact that citizens may change their votes after a deviation by a candidate that results in a configuration of positions that is not incentive compatible. This modification expands the set of possible equilibria, because it reduces the set of deviations that definitely make a candidate better off. We show, however (Proposition 2), that the core idea in our main result persists; in particular, in any equilibrium configuration there are at most two parties with more than two members.

Our model shows that economies of party size-even small economiesexert a powerful influence over the number of parties. Under our specific assumptions, the number of parties is limited to two. In Section 7 we discuss how this result is affected by variants of the model with a multidimensional policy space and uncertainty on the part of the candidates about their chances of election and about the citizens' preferences. In these variants, economies of party size remain potent, but result in the possibility of more than two parties coexisting in equilibrium.

\section{Relation With literature}

The literature on party-formation is large. One focus is Duverger's Law, which asserts that the number of parties is influenced by the electoral system. Duverger (1954, Book II, Ch. I) claims that "the simple-majority single-ballot system 
favours the two-party system" (p. 217) whereas "the simple-majority system with second ballot and proportional representation favour multi-partism" (p. 239).

Feddersen (1992) addresses the first part of the law. His result is driven by strategic voting by citizens, unlike ours, which is driven by the strategic maneuvering of candidates. Candidates, in fact, are absent from his model: citizens vote for positions, with positions that obtain many votes interpreted as parties. The outcome is a lottery among the positions that obtain the most votes. In an equilibrium, the winning positions are tied, so that any citizen, by changing her vote, can induce the election of a single candidate; if the citizens' payoff functions are concave then in the presence of more than two winning positions, some citizen can deviate and induce the certain election of a candidate whom she prefers to the lottery over the tied candidates. Thus in any equilibrium at most two positions receive votes. In our model, too, voters are pivotal in equilibrium. But this feature is not critical; in our model, configurations of the candidates' positions are ruled out as equilibria by moves by candidates, not by changes in the citizens' voting behavior. Our result does not depend on the nature of the citizens' preferences, but rather is driven by economies of party size. $^{4}$

The first part of Duverger's Law is given different theoretical support by Cox (1987) and Palfrey (1989), who formalize the idea that votes for candidates with little chance of winning are wasted, resulting in equilibria in which there are two candidates. This argument, like Feddersen's, rules out configurations as equilibrium on the basis of the citizens' incentives to change their votes rather than the candidates' incentives to change positions.

Morelli (2004) addresses both parts of Duverger's law. His model has elements in common with ours: parties decide whether to merge, and then face an election; the policy chosen by the resulting legislature is the median favorite position of the elected candidates. The considerations faced by a party deciding whether to change its position are also common to both models: how will

\footnotetext{
${ }^{4}$ Gerber and Ortuño-Ortín (1998) study a model related to Feddersen's, with a continuum of voters. They assume a continuous outcome function that weights parties by their sizes and gives proportionally larger weight to large parties. (Such a function is not consistent with a winnertakes-all electoral rule.) They show that a unique strong Nash equilibrium exists, in which there are two parties; the nature of the outcome function appears to play a key role in this result.
} 
the move affect the electoral chances of the party's candidates and how will the change in the resulting legislature affect the legislative outcome? But the fine structures of the models are very different. In our model, candidates are free to choose any position they wish. In Morelli's model, there are three districts, three possible positions, and exactly three potential parties. The leaders of the two extreme parties propose positions to the leader of the middle party, who can accept at most one of the proposals; no other changes in the parties' positions are permitted.

Morelli shows that if the distributions of the citizens' preferences are sufficiently similar in the three districts and sufficiently close to uniform within each district, then only one party is active under plurality rule whereas three parties are active under proportional representation. The basic argument is simple. First consider plurality rule. If voting is sincere, ${ }^{5}$ then except in the unlikely case that the two parties with the highest numbers of sincere votes are tied, there is a single winner in each district; given that running is costly, only one candidate therefore stands in each district. If the distributions of the citizens' preferences in the three districts are sufficiently similar, the single candidate in each district represents the same party. Now consider proportional representation. The number of votes for each party is the sum of its votes across the three districts. If the vote totals of the three parties do not differ too much, then proportional representation elects one candidate from each party; if the candidate is chosen randomly from the party's candidates in the three districts, then the probability of each candidate's becoming a member of the legislature is $\frac{1}{3}$, making it worthwhile for her to run. Thus when the districts do not differ too much, three parties are active under proportional representation. For preferences that are not sufficiently similar in the three districts, more that one party may be active under plurality rule, and in fact more parties may be active under plurality rule than under proportional representation.

This logic is very different from the reasoning behind our result. Morelli's one-party equilibria under plurality rule have no counterpart in our model because under our assumption of costly strategic voting, no one has an incentive to vote for a single party. The three-party equilibria do not exist in our model

\footnotetext{
${ }^{5}$ Morelli considers also a version of strategic voting in which abstention is not allowed.
} 
because a candidate in the smallest party has an incentive to deviate to one of the other parties, a move that does not affect the legislative outcome and reduces her cost; such an action is not unilaterally available to such a candidate in Morelli's model.

Several other ideas relating to party-formation that are more remote from the one that lies behind our model have been explored. Baron (1993) studies a model of proportional representation within the Hotelling-Downs framework. Citizens are not strategic, party formation is not costly, and the number of parties is fixed. Party size is determined by the fact that a large party has a diverse, and thus harder to please, membership, whereas such a party is more likely to be part of the government and be able to implement a policy appealing to its members.

Jackson and Moselle (2002) study a model of legislative bargaining in which legislators can benefit from forming parties that bind their members to cooperate with each other in the bargaining. In Snyder and Ting's (2002) model, parties are "brands" to imperfectly informed voters, aggregating ideologically similar candidates. Levy (2004) models the idea that political parties increase the ability of candidates to commit to policy positions. She finds that this increased ability affects party formation only when policies are multidimensional.

Finally, our model builds on that of Osborne et al. (2000). Their model can be interpreted as a simplified version of our game in which all candidates are automatically elected and economies of party size are absent. Under these assumptions, there is no cost-based incentive for individuals with different preferences to form parties.

\section{MOdEL}

Structure of game Our model is an extensive game. The set of players is the union of a finite set $P$ of politicians and a continuum $C$ of citizens. The set of possible policies is denoted $X$, which we assume to be the set of real numbers. ${ }^{6}$

\footnotetext{
${ }^{6}$ All our results hold also when $X$ is a completely ordered subset of a higher-dimensional set. (A set is completely ordered if its members are related by a complete, transitive, reflexive, antisymmetric ordering.)
} 
The game has two stages, in each of which actions are taken simultaneously. First, each politician decides whether to become a candidate, and if so which position to champion. Then each citizen chooses whether to vote, and if so for which candidate. The citizens' votes determine the candidates who are elected; the policy chosen by the legislature is the median of the policies championed by the elected candidates.

More precisely, the players' actions are specified as follows.

Politicians choose policies Each politician chooses either a member of $X$, meaning she is a candidate championing the policy she chooses, or the action $\theta$, meaning she is not a candidate.

Citizens vote Each citizen chooses either a candidate (i.e. a politician who chose a member of $X$ ), meaning she votes for that candidate, or the action $\theta$, meaning she does not vote.

A strategy for a politician is simply an action (a member of $X \cup\{\theta\}$ ). A strategy for a citizen is a function that associates with each action profile $a$ for the politicians either a politician $j$ for whom $a_{j} \in X$ (i.e. a vote for $j$ ) or $\theta$ (abstention). For a strategy profile $B$ of the citizens, we denote by $B(a)$ the action profile in the subgame following the history $a$ (the profile of citizens' votes when the politicians' positions are given by $a$ ) and by $B(a)(c)$ the action taken by citizen $c$ (the politician for whom $c$ votes, or $\theta$ if she does not vote) in this subgame.

Given the candidates' and citizens' strategies, the set of elected candidates is determined by an electoral rule. Rather than positing a specific rule, we adopt a formulation that encompasses a variety of rules that satisfy some natural conditions. Each rule in the class is defined by a quota function $Q: \mathbb{R}_{+}^{|P|} \rightarrow \mathbb{R}_{+}$that specifies the measure of votes a candidate needs to be elected. Precisely, for any profile $\alpha$ of vote totals for the candidates, a candidate is elected if and only if she obtains at least $Q(\alpha)$ votes. We assume that the function $Q$ is continuous, nondecreasing, and anonymous $\left(Q(\alpha)=Q\left(\alpha^{\prime}\right)\right.$ whenever $\alpha$ is a permutation of $\left.\alpha^{\prime}\right)$, and has the property that $Q(\alpha)=0$ if and only if $\alpha=0$.

The following electoral rules satisfy these assumptions.

First-past-the-post $Q(\alpha)=\max _{i \in P} \alpha_{i}$ (a candidate is elected if and only if she obtains at least as many votes as every other candidate). 
Hare quota $Q(\alpha)=\left(\sum_{i=1}^{n} \alpha_{i}\right) / \bar{k}$ for some number $\bar{k}$ (a candidate is elected if and only if she obtains at least the fraction $1 / \bar{k}$ of votes cast).

Fixed quota $Q(\alpha)=\delta$ (a candidate is elected if and only if she obtains at least the fixed amount $\delta$ of votes).

Note that the continuity assumption on $Q$ is consistent with a small change in the vote totals radically changing the set of elected candidates. For example, under the first-past-the-post rule, if two candidates are tied for the largest measure of votes, then they are both elected, whereas if candidate 1 obtains slightly more votes than candidate 2 , only candidate 1 is elected.

Of the assumptions we impose on an electoral rule, two are key: determinism and anonymity. These assumptions together imply that the number of elected candidates varies with the profile of vote totals. A rule that elects the same number, say $k$, of legislators for every profile of vote totals must select $k$ candidates when more than $k$ are tied for first place, and can do so only either randomly or non-anonymously. We discuss in Section 7.2 the implications of rules that elect a legislature of fixed size.

For any list $a$ of policies, we denote the median policy by $M(a)$, which we take to be the average of the left and right medians if the number of components of $a$ is even. ${ }^{7}$ If no politician is elected, the policy chosen is a fixed default policy $d$.

Payoffs Each politician cares about the policy chosen by the legislature and incurs a cost that is decreasing in the number of elected candidates who champion the same position as does she. More precisely, we assume that each politician $i$ has a valuation function $v_{i}$ over policies. When the policy chosen by the legislature is $x$ and her action is $a_{i}$, her payoff is

$$
\begin{cases}v_{i}(x) & \text { if } i \text { is not a candidate }\left(a_{i}=\theta\right) \\ v_{i}(x)-C_{p}(0) & \text { if } i \text { is a candidate }\left(a_{i} \in X\right) \text { but is not elected } \\ v_{i}(x)-C_{p}\left(N\left(a_{i}\right)\right) & \text { if } i \text { is a candidate and is elected }\end{cases}
$$

${ }^{7}$ All our results generalize to the case in which the policy chosen from a list with an even number is $S\left(a_{\ell}, a_{r}\right)$, where $a_{\ell}$ and $a_{r}$ are the left and right medians and $S$ satisfies $S(x, y)=S(y, x)$ and $x \leq S(x, y) \leq y$ whenever $x \leq y$. 
where $N\left(a_{i}\right)$ is the number of elected politicians who champion $a_{i}, C_{p}(k)>0$ for all $k$, and $C_{p}$ is a decreasing function for values of its argument at least equal to 1. The assumption that $C_{p}$ is decreasing means that there are economies of party size. Note that we impose no assumptions on the function $v_{i}$. Note also that we require no relation between $C_{p}(0)$ and $C_{p}(k)$ for $k \geq 1$; the relation between the cost incurred by an unelected candidate and the costs incurred by elected candidates plays no role in our analysis. Note also our assumption that the costs are shared only by elected candidates.

Citizens, like politicians, care about the policy chosen by the legislature. Each citizen incurs a cost if she votes. Specifically, citizen $c$ 's payoff when the policy chosen by the legislature is $x$ is

$$
\begin{cases}v(c, x) & \text { if } c \text { does not vote } \\ v(c, x)-C & \text { if } c \text { votes }\end{cases}
$$

where $C>0$. We impose no assumptions on $v$.

Define $u(c,(a, b))$ to be citizen $c$ 's payoff when the politicians' action profile is $a$ and the citizens' voting profile in the subgame following $a$ is $b$. (That is, $u(c,(a, b))$ is $v(c, x)$ if $c$ votes and $v(c, x)-C$ if she does not, where $x$ is the policy chosen by the legislature elected when the players' actions are given by $(a, b)$.)

Equilibrium Our solution concept is a variant of Nash equilibrium. In particular, we do not impose on an equilibrium the full force of subgame perfection. To understand our notion, first consider the definition of a Nash equilibrium.

A Nash equilibrium of our game is a pair $(a, B)$ consisting of a strategy profile $a$ for the politicians and a strategy profile $B$ for the citizens with the property that no politician can increase her payoff by changing her action, given the citizens' strategy profile $B$, and no voter can increase her payoff by changing her action when the politicians choose $a$ (i.e. $B(a)$ is a Nash equilibrium of the subgame following the history $a$ ).

An alternative form of this definition is useful. For any given strategy profile $B$ of the citizens, consider the strategic game in which the players are the politicians, each politician's set of actions is the same as it is in the whole game (i.e. 
$X \cup\{\theta\}$ ), and the outcome of any action profile $a$ is the policy chosen by the legislature elected when the citizens vote according to $B(a)$. Denote this strategic game by $G^{B}$. Then a Nash equilibrium of our (extensive) game is a strategy profile $(a, B)$ such that $a$ is a Nash equilibrium of $G^{B}$ and $B(a)$ is a Nash equilibrium of the subgame following $a$.

Our equilibrium notion differs from Nash equilibrium in two respects. First, instead of requiring that in each voting subgame no single citizen can profitably deviate, it requires that in each such subgame no small group of citizens voting for the same candidate can profitably deviate. Second, it requires that the citizens' votes do not change "unnecessarily" when any single politician changes her action in the first stage.

The first modification is a response to the insensitivity of the electoral outcome to a single citizen's action, given our assumption of a continuum of citizens. We formulate it precisely as follows. Consider the subgame following the politicians' action profile $a$. Denote by $b(c)$ the action of citizen $c$, which is either a candidate (i.e. a politician $j$ for whom $a_{j} \in X$ ) or $\theta$ (abstention). For any $\epsilon>0$ we say that a set of citizens is an " $\epsilon$-club" if it contains at most the fraction $\epsilon$ of the citizens and all members act in the same way (either they all vote for the same candidate, or none votes). More precisely, an $\epsilon$-club is a measurable set $S$ of citizens for which $0<\mu(S) \leq \epsilon$, where $\mu$ measures the size of a set of citizens, and for some action $j \in\left\{i \in P: a_{i} \in X\right\} \cup\{\theta\}$ we have $b(c)=j$ for every $c \in S .{ }^{8}$

DEFINITION 1. The voting profile $b$ in the subgame following the politicians' action profile $a$ is a small clubs Nash equilibrium (or simply an equilibrium) of the subgame if there exists $\epsilon>0$ such that for every $\epsilon$-club $S$ and every action $j \in\left\{i \in P: a_{i} \in X\right\} \cup\{\theta\}$ we have

$$
\int_{S} u(c,(a, b)) d \mu(c) \geq \int_{S} u\left(c,\left(a, b^{\prime}\right)\right) d \mu(c),
$$

where $b^{\prime}$ is the citizens' action profile that differs from $b$ only in that all members of $S$ take the action $j$.

\footnotetext{
${ }^{8}$ Note that if $C$ were finite and $\mu$ were the counting measure, the notion of a small clubs Nash equilibrium would coincide with the notion of a pure strategy Nash equilibrium.
} 
Given this definition of equilibrium in each subgame, the following definition is the appropriate variant of the notion of Nash equilibrium in the whole game.

Definition 2. A strategy profile $(a, B)$ is an equilibrium of the game if $a$ is a Nash equilibrium of the game $G^{B}$ and the voting profile $B(a)$ is a small clubs Nash equilibrium of the subgame following the history $a$.

Our second modification of the notion of Nash equilibrium is more substantial. Each voting subgame may have many Nash equilibria, with different sets of elected candidates. This multiplicity can support equilibria of the whole game in which a candidate is deterred from deviating in the first stage by an "unnecessary" change in the equilibrium of the subgame. That is, when the candidate deviates, the voting profile changes even though it remains an equilibrium and, if the citizens continue to adhere to it, the policy outcome remains the same.

Our notion of equilibrium rules out such "unnecessary" changes in the voting equilibrium. Precisely, let $(a, B)$ be an equilibrium of the whole game. Let $a_{i}^{\prime}$ be a deviation by politician $i$. If the voting equilibrium $B\left(a_{i}^{\prime}, a_{-i}\right)$ specified by $(a, B)$ for the subgame following the history $\left(a_{i}^{\prime}, a_{-i}\right)$ differs from $B(a)$ even though $B(a)$ is an equilibrium of this subgame and the policy outcome remains the same when the voters adhere to this voting profile, then we rule out $(a, B)$ as an equilibrium of the game. That is, we restrict attention to equilibria in which a deviation by a politician has no effect on the citizens' voting behavior if this voting behavior remains an equilibrium of the subgame reached after the deviation and, if the citizens adhere to this voting behavior, the policy outcome remains the same. We call such equilibria subgame persistent.

To define the notion of subgame persistent equilibrium precisely, say that the voting subgames following $a$ and $a^{\prime}$ are adjacent if $a$ and $a^{\prime}$ differ only in the action of a single candidate. Further, for any pair $(a, b)$ consisting of an action profile $a$ for the candidates and a voting profile $b$ for the citizens, define $A(a, b)$ to be the profile of positions of the elected candidates.

DEFINITION 3. An equilibrium strategy profile $(a, B)$ is a subgame persistent equilibrium of the game if $B\left(a^{\prime}\right)=B(a)$ whenever $a^{\prime}$ is adjacent to $a, B(a)$ is a 
small clubs Nash equilibrium of the subgame following $a^{\prime}$, and $M(A(a, B(a)))=$ $M\left(A\left(a^{\prime}, B(a)\right)\right)$.

Note that the definition does not restrict either the citizens' behavior in subgames not adjacent to $a$ or in subgames for which $B(a)$ is not a small clubs Nash equilibrium.

To return to the examples discussed earlier, the eight-candidate example on page 5 is not a subgame persistent equilibrium because when a candidate championing the far-left position moves to the center-left position the voting profile remains an equilibrium, the outcome remains the same if the citizens continue to adhere to this profile, and the candidate who moves leaves a two-member party to become a member of a three-member party. By contrast, the following three-candidate example may be a subgame persistent equilibrium for some specifications of the players' preferences, because any move by a candidate disrupts the voting equilibrium and may result in the candidate's not being elected.

\section{MAIN RESUlT}

Our main result is that in any subgame persistent equilibrium in which some citizens vote, there are exactly two parties and possibly a small number of independents.

To state the result precisely, define a strategy profile $(a, B)$ to have positive voter turnout if a positive measure of citizens vote on the equilibrium path (i.e. it is not the case that $B(a)(c)=\theta$ for almost all $c$ ). Note that requiring positive voter turnout does not restrict turnout in subgames reached when politicians deviate from their equilibrium actions. If two or more politicians choose the same position $x \in X$, we say that $x$ is a party; if the position $x$ is chosen by a single politician, we say that $x$ is an independent.

PROPOSITION 1. In a subgame persistent equilibrium with positive voter turnout, the number of parties is at most two.

- If there are two parties, then the sizes of the parties differ by at most one and there are at most three independents, whose positions lie between the positions of the parties. 
- If there is one party, then there are at least as many independents as members of the party and the positions of all independents are on the same side of the party.

Proofs of this result and of Proposition 2 are given in the Appendix. Note that the result gives only necessary conditions for equilibrium, independently of the candidates' and citizens' preferences.

We show that for any configuration of positions that does not satisfy the conditions in the proposition, a candidate can either withdraw or move to a larger party without affecting the median position of the elected candidates, in both cases increasing her payoff. To show that a move does not affect the median position of the elected candidates, we need to consider its effect on the citizens' voting behavior. Our technique is to restrict attention to moves for which the voting behavior remains an equilibrium, so that subgame persistence implies that this behavior does not change. We show (Step 2 of the proof) that a move by a candidate to a position currently occupied by at least two candidates has this property if it (i) does not affect the median position of the candidates and (ii) would not affect this median position even if any one of the other candidates were to switch to nonparticipation.

Denote the profile of the candidates' positions by $a$ and the subgame following any profile $\bar{a}$ by $\Gamma(\bar{a})$. Consider a move of candidate $i$ from $a_{i}$ to a position $x$ occupied by at least two candidates. To show that if such a move satisfies (i) and (ii) then the voting profile remains an equilibrium, we argue that for every change in the voting behavior of a small group of citizens in the subgame $\Gamma\left(x, a_{-i}\right)$ following $i$ 's deviation there is a change in the voting behavior of this group in the subgame $\Gamma(a)$ that induces the same outcome. Condition (ii) has the following role. Suppose that a small group of citizens voting for some candidate $j \neq i$ deviates to abstention. This deviation must change the set of elected candidates to $E \backslash\{j\}$, where $E$ is the original set of elected candidates. (If it did not affect the set of elected candidates, it would be a profitable deviation in $\Gamma(a)$, given that voting is costly.) Thus it changes the outcome from the median of the positions of the candidates in $E$ to the median of the positions of the candidates in $E \backslash\{j\}$. In $\Gamma(a)$, the outcome thus changes from $M(a)$ to $M\left(\theta, a_{-j}\right)$, whereas 
in $\Gamma\left(x, a_{-i}\right)$ it changes from $M\left(x, a_{-i}\right)$ to $M\left(\theta,\left(x, a_{-i}\right)_{-j}\right)$. Now, $M(a)=M\left(x, a_{-i}\right)$ by (i), so in order for the change in voting behavior to have the same effect on the outcome in both subgames we need $M\left(\theta, a_{-j}\right)=M\left(\theta,\left(x, a_{-i}\right)_{-j}\right)$, which is condition (ii).

To illustrate this point, the eight-candidate example considered on page 5 satisfies (ii), but the move of the left candidate to the middle position in the three-candidate example considered in the following paragraph does not. Specifically, if in the latter example the right candidate is absent, the move of the left candidate affects the outcome, changing it from the average of the positions of the left and middle candidates to the position of the middle candidate. Thus we know that the move of the left candidate in the eight-candidate example is profitable, whereas we cannot make the same inference for the move of the left candidate in the three-candidate example.

\section{Post-election maneuvering}

After a candidate is elected, she may be able to affect the policy chosen by the legislature by championing a policy different from the one she proposed when elected. (Real politicians have been known to engage in such maneuvers.) Further, citizens may build this possibility into their calculations when voting: they may be reluctant to vote for a candidate who has an incentive to change positions after the election. If voters reason in this way, pre-election position changes by candidates that do not affect voting behavior under the previous assumptions may cause citizens to change their voting behavior, reducing the set of deviations by candidates that are necessarily profitable and thus expanding the set of configurations that are equilibria. We show that indeed the set of possible equilibrium party configurations expands. But the expansion is minor: parties containing two members, in addition to independents, may take positions in the middle of the spectrum; otherwise the characteristics of the equilibria are unchanged.

To illustrate the issue, consider the configuration of the candidates' positions shown in the left panel of Figure 2, in which two parties, each with two members, lie between two larger parties, each with three members. Assume all candidates obtain the same number of votes and are elected. Under our previous assump- 

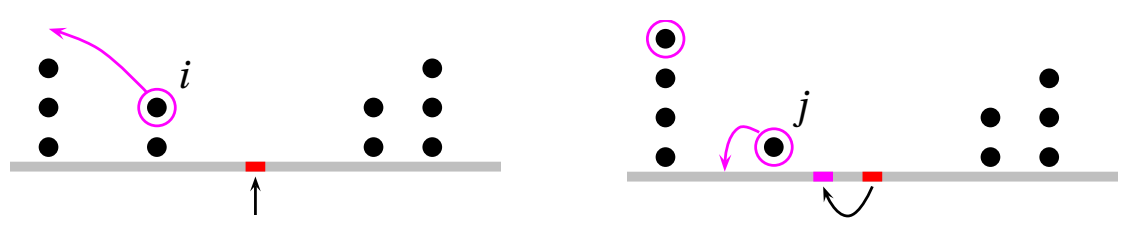

FIGURE 2. An example in which a candidate's incentives change when another candidate changes positions.

tions, this configuration is not the outcome of a subgame persistent equilibrium. If candidate $i$, one of the members of the center-left party, moves to the left party, then the median position of the elected legislators remains the same if all candidates remain elected; further, this move does not affect the median position of the legislators if any one candidate is absent from the game. Thus the citizens' voting behavior remains an equilibrium of the subgame reached after the candidate changes positions, so that the candidate's move is profitable and thus the configuration is not consistent with subgame persistent equilibrium. However, after $i$ 's move, changes in the position of the remaining member $j$ of the centerleft party affect the legislators' median position in ways that they did not before. For example, if, after $i$ 's move, $j$ moves to the left (see the right panel of the figure), the median legislators' position moves to the left, whereas before $i$ 's move such a move on the part of $j$ had no effect on the median position. Thus citizens may fear that $i$ 's move will lead to a change in $j$ 's position after the election, and thus may wish to change their votes when $i$ moves, even though, given the other candidates' positions, their behavior remains an equilibrium of the voting subgame. Consequently, the original configuration, in the left panel of Figure 2, may be stable: $i$ 's move may precipitate a change in the voting equilibrium and this change may make $i$ worse off (for example, she may no longer be elected). Candidate $j$ does not necessarily have an incentive to change positions after $i$ moves (her incentive depends on her payoff function), but it is possible that she does, so we cannot rule out the original configuration as an equilibrium.

This example depends, however, on the center-left party having exactly two members. If this party has three or more members, then $i$ 's move does not affect the ability of the other members of the party to alter the outcome by changing their positions. Thus if the center-left party has three or more members, 
the move by $i$ is profitable, and the configuration is not an equilibrium. Consequently, although the requirement that a candidate's move not give new incentives for the other candidates to change their positions admits new configurations as equilibrium outcomes, it does not admit many such new configurations.

To state our result formally, we first define a pair consisting of an action profile $a$ for the candidates and a voting profile $b$ for the citizens in the subgame following $a$ to be incentive compatible if no elected candidate can increase her payoff by changing her position after being elected.

DEFINITION 4. Let $a$ be an action profile for the candidates and let $b$ be a voting profile for the citizens in the subgame following $a$. The pair $(a, b)$ is incentive compatible if for any policy $x \in X$, no elected legislator $i$ prefers the legislative outcome when her position is $x$ to the legislative outcome when her position is $a_{i}$, given the other legislators' positions (as determined by $(a, b)$ ). That is,

$$
v_{i}(M(A(a, b))) \geq v_{i}\left(M\left(x, A_{-i}(a, b)\right)\right) \text { for every } x \in X,
$$

where $A(a, b)$ is the profile of the elected candidates' positions (and $v_{i}$ is candidate $i$ 's valuation function).

We now define an equilibrium strategy profile $(a, B)$ to be a "subgame ICpersistent equilibrium" if (a) the equilibrium outcome is incentive compatible and (b) the citizens' voting behavior in the subgame following a deviation by any candidate is $B(a)$ if this behavior is an equilibrium of the subgame, the policy outcome remains the same if the citizens adhere to this voting profile, and the outcome in the subgame is incentive compatible. Condition (a) potentially reduces the set of equilibria. However, because we impose no restrictions on the legislators' payoff functions, the condition does not imply any restrictions on the character of the equilibrium configurations. The power of the definition comes from condition (b), which expands the set of equilibria because it requires the citizens' voting behavior to remain the same after a candidate's deviation only if the outcome in the resulting subgame is incentive compatible.

DEFINITION 5. An equilibrium strategy profile $(a, B)$ is a subgame IC-persistent equilibrium of the game if 
- $(a, B(a))$ is incentive compatible

- $B\left(a^{\prime}\right)=B(a)$ if $a^{\prime}$ is adjacent to $a, B(a)$ is a small clubs Nash equilibrium of the subgame following $a^{\prime}, M(A(a, B(a)))=M\left(A\left(a^{\prime}, B(a)\right)\right)$, and $\left(a^{\prime}, B(a)\right)$ is incentive compatible.

To state our result, call a party with more than two members a large party and one with exactly two members a small party.

PROPOSITION 2. In a subgame IC-persistent equilibrium with positive voter turnout, the number of large parties is at most two.

- If there are two large parties, then the sizes of the parties differ by at most two and there are either four additional candidates who form two small parties, three additional candidates who form one small party and an independent, two additional candidates who form a small party, or up to three independents; the positions of the additional candidates lie between the positions of the large parties.

- If there is one large party, then there are at least as many independents as members of the party and the positions of all independents are on the same side of the large party; there may be a small party between the independents and the large party.

- If there are no large parties, then all candidates are independents.

This result says that in addition to the configurations identified in Proposition 1 , some configurations in which up to two small parties lie between the two large parties are possible in a subgame IC-persistent equilibrium.

\section{EXISTENCE OF AN EQUILIBRIUM}

A subgame persistent equilibrium exists under weak conditions. Assume that the distribution of the citizens' favorite positions has a unique median, denoted $m$. Assume that the payoff function of each politician is single-peaked and for some number $n \geq 2$, the favorite positions of at least $n$ politicians are less than $m$ and the favorite positions of at least $n$ politicians are greater than $m$. Finally, 
make two assumptions about the quota function. Suppose that initially every candidate receives the same fraction of the votes. Assume that if the members of a small group of citizens voting for one of the candidates, say $j$, switch their votes to another candidate, then the quota does not increase (so that if all candidates were elected initially, them all with the possible exception of $j$ remain elected). Assume also that if a small group of citizens voting for one of the candidates, say $j$, switches to abstention, the quota falls by less than the size of the group of deviants, so that $j$ is no longer elected.

If, in such an environment, the costs of running as a candidate in an $n$ member party and of voting are small enough (precise bounds are given subsequently), for $\delta>0$ small enough the game has a subgame persistent equilibrium in which $n$ politicians with favorite positions less than $m$ choose the position $m-\delta, n$ politicians with favorite positions greater than $m$ choose the position $m+\delta$, and the remaining politicians choose nonparticipation. When the politicians take these actions, all citizens vote for a candidate whose position is closest to their favorite position, with the votes equally divided between the candidates in each party. In every other subgame each citizen votes for the same politician as she does in this subgame if the politician is a candidate, and otherwise abstains.

Denote this strategy profile by $\left(a^{*}, B^{*}\right)$. To find conditions under which $\left(a^{*}, B^{*}\right)$ is a subgame persistent equilibrium, consider each type of player in turn.

Politicians A politician who is a candidate remains elected if she changes positions, but moves the outcome further from her favorite position and hence is worse off (given that her payoff function is single-peaked). (She can move the outcome only to some point in $[m, m+\delta]$ if she is in the left party and only to some point in $[m-\delta, m]$ if she is in the right party.) A politician who is a candidate causes the outcome to change to the position of the other party if she withdraws. Thus a politician $i$ whose favorite position is less than $m$ does not want to withdraw if $v_{i}(m)-C_{p}(n) \geq v_{i}(m+\delta)$, or $C_{p}(n) \leq v_{i}(m)-v_{i}(m+\delta)$. Similarly a politician $i$ whose favorite position is greater than $m$ does not want to withdraw if $C_{p}(n) \leq v_{i}(m)-v_{i}(m-\delta)$. A politician who is not a candidate is not elected if she becomes a candidate, so does not affect the outcome. 
Citizens If, in the subgame following $a^{*}$, the members of a small group of citizens voting for some candidate $j$ switch their votes to another candidate, either all candidates remain elected or all with the exception of $j$ are elected (by our assumption on the quota function), so that the citizens are not better off.

If, in the subgame following $a^{*}$, the members of a small group of citizens voting for a candidate in the left party switch to abstention, then that candidate is no longer elected (by our assumption on the quota function), so that the outcome changes from $m$ to $m+\delta$. For this deviation not to be profitable, we need $v(c, m)-C \geq v(c, m+\delta)$, or $C \leq v(c, m)-v(c, m+\delta)$ for every citizen $c$ whose favorite position is less than $m$. Symmetrically, considering a small group of citizens voting for a candidate in the right party leads to the condition $C \leq v(c, m)-v(c, m-\delta)$ for every citizen $c$ whose favorite position is greater than $m$. If these conditions are satisfied, the voting profile $B\left(a^{*}\right)$ is a Nash equilibrium of the subgame following $a^{*}$. For any $a^{\prime}$ adjacent to $a^{*}$, we have $B\left(a^{\prime}\right)=B\left(a^{*}\right)$ (by the definition of $B$ ), so the profile satisfies the condition for subgame persistence.

In summary, in the environment described in the first paragraph of this section, if $C_{p}(n) \leq v_{i}(m)-v_{i}(m+\delta)$ for every politician whose favorite position is less than $m, C_{p}(n) \leq v_{i}(m)-v_{i}(m-\delta)$ for every politician whose favorite position is greater than $m, C \leq v(c, m)-v(c, m+\delta)$ for every citizen $c$ whose favorite position is less than $m$, and $C \leq v(c, m)-v(c, m-\delta)$ for every citizen $c$ whose favorite position is greater than $m$, then the strategy profile is a subgame persistent equilibrium. For any fixed value of $\delta$, these conditions are satisfied if $C_{p}(n)$ and $C$ are small enough.

Our assumption that the politicians' payoff functions are single-peaked is mild, but is stronger than necessary: we need only that $v_{i}(x) \leq v_{i}(m)$ for every $x \in[m, m+\delta]$ for a politician whose favorite position is less than $m$, and symmetrically for a politician whose favorite position is greater than $m$. 


\section{AMELIORATING FACTORS}

Our results show that the presence of economies of party size in a static deterministic environment with a one-dimensional policy space dramatically limit the equilibrium configurations of candidates' positions to ones in which there are at most two parties. We now explore how deviations from this environment affect this conclusion.

\subsection{Multidimensional policy space}

To extend the model to a multidimensional policy space we need to find a substitute for the median. For a one-dimensional policy space, many models of legislative decision-making generate the median of the legislators' positions as the outcome. For a multidimensional policy space, no outcome has the same theoretical support. We consider a simple generalization of the median: the component-wise median. That is, we assume that if the positions the legislators champion are given by the points $x_{1}, \ldots, x_{k}$ where $x_{i} \in \mathbb{R}^{\ell}$ for $i=1, \ldots, k$ (and $\ell \geq 2$ ), then for each $j=1, \ldots, \ell$, the $j$ th component of the outcome is the median of the $j$ th components of $x_{1}, \ldots, x_{k}$.

Under this assumption, the incentive to economize on the cost of getting elected drives candidates to agglomerate in much the same way as it does in the one-dimensional model. The number of parties that emerge, however, is larger. Consider the case of a two-dimensional policy space. Suppose that the candidates' positions are the black disks shown in Figure 3. Then the componentwise median is given by the red disk at the intersection of the horizontal and vertical lines. Suppose that candidate $i$ moves to the position of candidate $j$. Then the outcome remains the same: $i$ 's move does not affect the median in either direction. Thus if $i$ remains elected, the move is profitable and the original configuration is not an equilibrium. Not only does $i$ 's move not affect the median in either direction, but this move would not affect the median in either direction even if one of the other candidates were not elected. Thus by the same logic as in the case of a one-dimensional policy space, the citizens' voting profile for the original positions remains an equilibrium after $i$ moves, so that under subgame persistence, $i$ remains elected. Thus $i$ 's move is profitable, and hence 


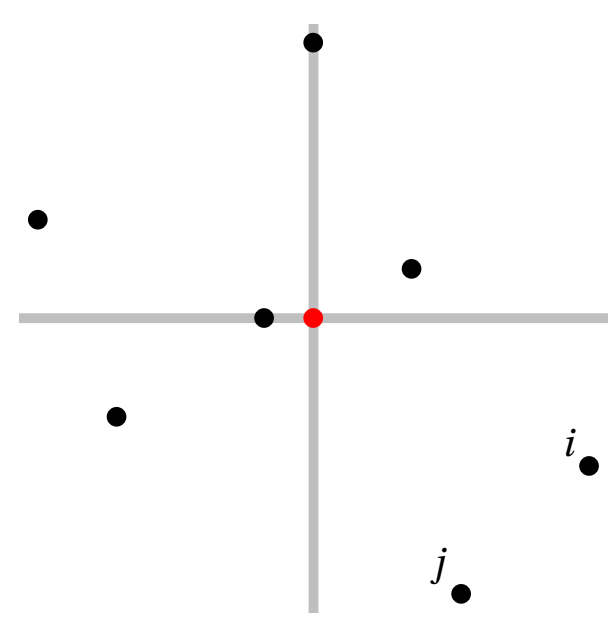

FIGURE 3. Candidates' positions in a two-dimensional policy space.

the configuration of positions is not an equilibrium.

This example suggests that the forces of agglomeration in a multidimensional policy space resemble those in a one-dimensional space, although the number of parties to which they lead is greater than two. Specifically, the example suggests that in a two-dimensional space, candidates in the same quadrant can profitably agglomerate, so that no equilibrium has more than four parties with more than two members. Extrapolation from the example suggests that in $k$ dimensions, the number of parties with more than two members is at most $2^{k}$.

\subsection{Randomness in tie-breaking}

We have assumed that if several candidates tie for the highest number of votes, they are all elected. An alternative assumption is that a fixed number of candidates is elected, with ties broken randomly. As in the deterministic case, all candidates receive the same number of votes in any equilibrium. Assume that the cost of running a party is shared by all the candidates in the party who are tied for first place, regardless of whether they are among the candidates randomly chosen to be members of the legislature. Then an argument like the one for the deterministic case shows that the two most extreme parties on the left must have at least $\frac{1}{2}(k-1)$ members if $k$ is odd and at least $\frac{1}{2} k$ members if $k$ is even, where $k$ is the size of the legislature, and the same for the two most extreme parties on the right. The reason is that if the number of members of the 
two most extreme parties on one side or the other is smaller than this number, then a member of either of these parties can switch to the other party without affecting the legislative outcome, regardless of the (randomly-determined) composition of the legislature.

These conditions do not limit the number of parties to two: in this case, economies of party size alone do not rule out the existence of more than two parties. However, they do imply that the "fringe" parties are large relative to the size of the legislature. Further, this result, like our results in the deterministic case, holds for any payoff functions; for specific payoff functions we may be able to narrow down the set of equilibria further. The reason that, independently of the payoff functions, we cannot rule out configurations in which the fringe parties are large is that with positive probability the legislature consists exclusively of members of these parties, in which case a move between them may affect the position of the median legislator. If the number of candidates relative to the size of the legislature is large, however, this probability is small, and for many payoff functions any loss a candidate suffers because her move to a larger party changes the median adversely is more than offset by the gain she obtains when the legislature does not consist exclusively of extreme parties, so that in fact such configurations can be ruled out as equilibria.

In summary, when a fixed-size legislature is selected by breaking ties randomly, the existence of economies of party size does not limit the number of parties independently of the candidates' and citizens' payoff functions, as it does when the electoral rule is deterministic. However, the logic underlying the anal-

ysis of the deterministic case still holds and puts significant restrictions on the possible equilibrium configurations of the candidates' positions.

\subsection{Multi-district systems}

Suppose that citizens are divided among electoral districts, in each of which there is a set of candidates. Each citizen casts a vote for one of the candidates in her district. Assume that for each district there is a separate quota function; the candidates elected in any given district are the ones who obtain at least the quota of votes for that district, with the quota depending on the profile of votes in the district. As before, assume that citizens and politicians care only about the 
final legislative outcome, which is the median position of the candidates elected in all districts.

If candidates can move between districts, a key element in our earlier argument fails: such moves must affect the voting profile (because citizens vote only for candidates in their district), so we cannot deduce that they do not affect the set of elected candidates. Because of the possible multiplicity of equilibria in the voting subgames, the failure of this argument suggests that the set of equilibria of the whole game is much larger than it is in the original model. As in the original model, some of these equilibria rely on implausible changes in voting behavior in response to changes in the candidates' positions, but the notion of subgame persistent equilibrium does not eliminate such equilibria; a different approach is required to isolate "sensible" equilibria.

If each candidate can move only within her district, then the incentives in our original model tend to limit the number of parties within each district to two. However, the positions of the parties may differ between districts, so that more than two parties may exist in the society as a whole. For example, if there are two candidates in district 1 , at the positions $w$ and $z$, and two candidates in district 2, at the positions $x$ and $y$ with $w<x<y<z$, then no candidate can necessarily profitably move to a different position in her district. The obvious move that would be profitable in the original model if it did not affect the voting equilibrium, from $w$ to $x$, is not possible, because these two positions are in different districts.

In summary, our model is not well-suited to study the (reasonable?) case in which candidates can move between districts, and the number of parties possible in an equilibrium exceeds two if each candidate is restricted to moves within her own district.

\subsection{Uncertain future}

Our model is static. To add a dynamic dimension to it, we need to decide the penalty, if any, that a candidate incurs if she changes her positions from one period to the next. If this penalty is large enough to cause a candidate to pick a single fixed position for all periods, then when contemplating the position to choose she needs to consider the possibility that even if a move does not affect 
the legislative outcome given the current set of candidates, it may do so for some future set of candidates (who may enter in response to changes in the citizens' preferences, for example). This consideration may lead a candidate to choose a position different from the one she would choose in a static environment. ${ }^{9}$

To give a specific example, a far-left candidate may, in a static environment, want to move to a center-left party, but may refrain from doing so in an environment in which the possibility exists of a significant shift in voters' preferences that would put her current position at the center of political spectrum. The incentives that drive our main result are present in such a model, but the greater the possible variation in citizens' preferences, the less significant they are.

\section{CONCLUSiON}

Economies of scale in a model of static competition between firms leads to the emergence of a monopolist. We have shown that economies of party size in a static deterministic model of single-issue electoral competition leads to the emergence of at most two parties, of approximately the same size. This result is robust to post-election maneuvering, but depends on the existence of a single issue and is diluted by randomness in the electoral rule, by the presence of forward-looking politicians when future changes in the citizens' preferences are possible, and by the existence of a multi-district system.

Our model is stripped of any dynamic elements in order to isolate the role of economies of party size. Given that the environment we study is static, we define a "party" simply as a collection of candidates championing the same position. Actual parties operate in a much richer, dynamic environment; they are uncertain about the issues they will confront, the citizens' preferences, and the parties with which they will compete. To capture at least some aspects of such an environment requires a model different from the ours, in which a party should probably be defined as a long-lived organization.

Our notion of equilibrium is a variant of Nash equilibrium; we consider only deviations by single candidates and small groups of citizens taking the same action. We do not consider deviations by groups of candidates, and in particu-

\footnotetext{
${ }^{9}$ We thank a referee for bringing this point to our attention and for providing the example in the next paragraph.
} 
lar do not allow all the members of a party to simultaneously change positions, thereby moving the party's position. Simply adding coalitional deviations to the list of possible deviations would reduce the set of equilibria (thus preserving our result that there are at most two parties). The effect of restricting all deviations, including ones by a single candidate, to be credible (in the sense of coalition proof Nash equilibrium, for example), is unclear.

\section{APPENDIX: PROOFS}

We first establish some properties of an equilibrium of a voting subgame.

LEMMA 1. In a (small clubs) Nash equilibrium of a voting subgame in which a positive measure of citizens votes, every elected candidate obtains the same measure of votes, equal to the quota, and no unelected candidate obtains any votes.

PROOF. Let $a$ be profile of the candidates' actions and let $b$ be an equilibrium voting profile of the citizens in the subgame $\Gamma(a)$ following $a$. Suppose that a positive measure of citizens votes in $b$. Given our assumption on the quota function that the quota is zero if and only if the measure of votes is zero, the quota in the equilibrium is positive. Denote the quota by $q$.

We first argue that no unelected candidate obtains any votes. Suppose to the contrary that candidate $i$ is not elected and obtains a positive measure of votes. We argue that a small set of the citizens voting for $i$ can switch to not voting without affecting the outcome, thus increasing their payoffs. Let $\delta$ be the largest measure of votes received by any unelected candidate, so that $\delta \in(0, q)$. Suppose that $\epsilon>0$ and an $\epsilon$-club voting for candidate $i$ switches to not voting. Because the quota function is nondecreasing, the quota does not increase; because it is continuous, for $\epsilon$ small enough the quota remains greater than $\delta$. Thus the set of elected candidates, and hence the policy outcome, remain the same. The members of the $\epsilon$-club save the cost of voting, contradicting the fact that $b$ is an equilibrium of $\Gamma(a)$. Thus no unelected candidate receives votes.

We now argue that every elected candidate obtains the same number of votes, equal to the quota. Suppose to the contrary that candidate $i$ is elected and obtains more than the quota of votes. By the same argument as in the previous 
paragraph, a deviation to nonvoting by a sufficiently small $\epsilon$-club voting for candidate $i$ does not affect the set of elected candidates and hence does not affect the policy outcome, so that the members of the $\epsilon$-club are better off, contradicting the fact that $b$ is an equilibrium of $\Gamma(a)$. Thus no candidate obtains more votes than the quota.

We now show that when an unelected candidate withdraws, the citizens' voting behavior remains an equilibrium.

LEMMA 2. If candidate $i$ is not elected in a Nash equilibrium $b$ of the voting subgame $\Gamma(a)$ following $a$ in which a positive measure of citizens votes, then $b$ is an equilibrium of the voting subgame $\Gamma\left(\theta, a_{-i}\right)$ following $\left(\theta, a_{-i}\right)$.

PROOF. Any deviation by a group of voters from the voting profile $b$ in $\Gamma\left(\theta, a_{-i}\right)$ leads to the same voting profile, and hence the same quota, as does the same deviation in $\Gamma(a)$. Thus such a deviation can lead to different sets of elected candidates in the two subgames only if it causes candidate $i$ to be elected in $\Gamma(a)$. By Lemma 1, candidate $i$ receives no votes in $b$, so for her to be elected requires the deviation to reduce the quota to 0 . But the quota is originally positive and the quota function is continuous, so for a sufficiently small value of $\epsilon$, no deviation by an $\epsilon$-club reduces the quota to zero. Thus any deviation by a sufficiently small $\epsilon$-club generates the same change in the set of elected candidates in $\Gamma(a)$ as it does in $\Gamma\left(\theta, a_{-i}\right)$. Since $b$ is an equilibrium of $\Gamma(a)$, it is thus an equilibrium of $\Gamma\left(\theta, a_{-i}\right)$.

The next result shows that the policy outcome changes when a candidate withdraws, holding constant the remaining candidates' actions. (If it did not change, then citizens could stop voting for the candidate without affecting the outcome.)

LEMMA 3. If candidate $i$ is elected in a Nash equilibrium of the subgame $\Gamma(a)$ following $a$ in which a positive measure of citizens votes, then $M\left(\theta, a_{-i}\right) \neq M(a)$.

PROOF. Suppose that the members of a small club of citizens voting for $i$ switch to nonparticipation. Then the quota does not increase (by our assumption that the quota function is nondecreasing), so that either all candidates remain elected, 
in which case the policy outcome remains the same, or all with the exception of $i$ are elected. If all candidates remain elected or all with the exception of $i$ are elected and $M\left(\theta, a_{-i}\right)=M(a)$, then the policy outcome remains the same, so that given that the cost of voting is positive, the members of the club are better off and hence the voting profile is not an equilibrium of the subgame $\Gamma(a)$. Hence all with the exception of $i$ are elected and $M\left(\theta, a_{-i}\right) \neq M(a)$.

We are now ready to prove Propositions 1 and 2.

Proof of Proposition 1. Let $(a, B)$ be a subgame persistent equilibrium with positive voter turnout. We first argue that every candidate is elected, because an unelected candidate who switches to nonparticipation does not affect the set of elected candidates, and hence increases her payoff.

STEP 1. In $(a, B)$, every candidate is elected.

Proof. Suppose that in the equilibrium $(a, B)$, candidate $i$ is not elected. Then by Lemma 2, $B(a)$ is an equilibrium of the subgame $\Gamma\left(\theta, a_{-i}\right)$ following $\left(\theta, a_{-i}\right)$. Hence by subgame persistence, the voting profile $B\left(\theta, a_{-i}\right)$ in this subgame is equal to $B(a)$, so that the policy outcomes in the two subgames are the same.

Candidate $i$ 's deviation to nonparticipation reduces her costs; because it does not change the policy outcome, it is profitable, contradicting the fact that $(a, B)$ is an equilibrium. Hence every candidate is elected.

Now suppose that candidate $i$ deviates to a position $x$ that is occupied by at least two other candidates. We argue that $B(a)$ remains an equilibrium of the resulting subgame if (a) the policy outcome is not affected and (b) the policy outcome would not be affected even if any one of the other candidates were absent from the election, in both cases assuming that all candidates are elected. Given this conclusion, under subgame persistence $B(a)$ is the voting profile in the resulting subgame and hence candidate $i$ remains elected.

Precisely, conditions (a) and (b) are

$$
\begin{gathered}
M(a)=M\left(x, a_{-i}\right) \\
M\left(\theta, a_{-j}\right)=M\left(\theta,\left(x, a_{-i}\right)_{-j}\right) \text { for all } j \neq i .
\end{gathered}
$$


STEP 2. If $x$ satisfies (1) and (2) for some candidate $i$ and $\left\{j \in P: a_{j}=x\right\} \geq 2$, then $B(a)$, the voting equilibrium of the subgame $\Gamma(a)$ following $a$ in the equilibrium $(a, B)$, is also an equilibrium of the subgame $\Gamma\left(x, a_{-i}\right)$ following $\left(x, a_{-i}\right)$ (in which candidate $i$ deviates to $x)$, so that $B\left(x, a_{-i}\right)=B(a)$ and in particular $i$ remains elected in $\Gamma\left(x, a_{-i}\right)$.

Proof. Suppose that the citizens use the strategy profile $B(a)$ in the subgame $\Gamma\left(x, a_{-i}\right)$ that is reached if candidate $i$ deviates to $x$ from $a_{i}$. Then the set of elected candidates in this subgame is that same as it is in $\Gamma(a)$. (Remember that each citizen votes for a candidate, not a position.) By Step 1, all candidates are elected in $\Gamma(a)$, so the policy outcome in $\Gamma(a)$ is $M(a)$ and all candidates are elected in $\Gamma\left(x, a_{-i}\right)$, so that the policy outcome in $\Gamma\left(x, a_{-i}\right)$ is $M\left(x, a_{-i}\right)$. Thus by (1), the policy outcomes in the two subgames, $M(A(a, B(a)))$ and $M\left(A\left(\left(x, a_{-i}\right), B(a)\right)\right)$, are the same.

We now argue that $B(a)$ is an equilibrium of $\Gamma\left(x, a_{-i}\right)$. We do so by showing that for each possible deviation by an $\epsilon$-club in $\Gamma\left(x, a_{-i}\right)$, there is a deviation by the same $\epsilon$-club in $\Gamma(a)$ that has the same effect on the policy outcome. In all but one case, the deviation with the same effect in $\Gamma(a)$ is exactly the same as the deviation in $\Gamma\left(x, a_{-i}\right)$.

We begin by determining the effect on the set of elected candidates of each possible deviation from $B(a)$ (in either subgame). Denote by $E$ the set of elected candidates under $B(a)$.

Deviation by $\epsilon$-club voting for some candidate $j$ : If the club deviates to nonparticipation, the set of elected candidates changes to $E \backslash\{j\}$. (It cannot remain $E$, because the deviating club would then be unambiguously better off.)

If the club deviates to vote for some candidate $j^{\prime} \neq j$, the set of elected candidates remains $E$, changes to $\varnothing$ (no candidates are elected), changes to $E \backslash\{j\}$, or changes to $\left\{j^{\prime}\right\}$ (depending on the nature of the quota function).

Deviation by nonvoting $\epsilon$-club: If the club deviates to vote for some candidate $j^{\prime}$, the set of elected candidates remains $E$, changes to $\varnothing$, or changes to 
$\left\{j^{\prime}\right\}$ (depending on the quota function).

For each possible set of elected candidates after a deviation, we argue as follows.

$E$ : The policy outcome in both subgames remains $M(a)=M\left(x, a_{-i}\right)$.

$E \backslash\{j\}$ with $j \neq i$ : The policy outcome in $\Gamma(a)$ changes to $M\left(\theta, a_{-j}\right)$ and the policy outcome in $\Gamma\left(x, a_{-i}\right)$ changes to $M\left(\theta,\left(x, a_{-i}\right)_{-j}\right)$. These two outcomes are the same by (2).

$\left\{j^{\prime}\right\}$ where $j^{\prime} \neq i$ : The policy outcome in both subgames changes to $a_{j^{\prime}}$.

$\varnothing$ : The policy outcome in both subgames changes to $d$ (the default policy).

$E \backslash\{i\}$ : The policy outcome in both subgames changes to $M\left(\theta, a_{-i}\right)$.

$\{i\}$ : In this case, the policy outcome induced by the deviation in $\Gamma(a)$, namely $a_{i}$, differs from the policy outcome induced by the deviation in $\Gamma\left(x, a_{-i}\right)$, namely $x$. However, another deviation by the same $\epsilon$-club in $\Gamma(a)$ induces the policy outcome $x$ : rather than deviating to vote for $i$, the members of the $\epsilon$-club deviate to vote for a candidate, say $j$, whose position is $x$ (the existence of which is guaranteed by our assumption that at least two candidates take the position $x$ ). Given that $i$ alone is elected when the club deviates to vote for $i$ (instead of either voting for some other candidate or not voting), by the anonymity of the quota function, $j$ alone is elected when the club deviates to vote for $j$, generating the policy outcome $x$. Because $B(a)$ is an equilibrium of $\Gamma(a)$, this deviation does not make the $\epsilon$ club better off. Thus the deviation to vote for $i$ by the same $\epsilon$-club in the subgame $\Gamma\left(x, a_{-i}\right)$, which also generates the policy outcome $x$, is not profitable.

We conclude that $B(a)$ is a small clubs Nash equilibrium of $\Gamma\left(x, a_{-i}\right)$. Thus $B\left(x, a_{-i}\right)=B(a)$ from the definition of subgame persistence. Hence by Step 1, all candidates are elected in $\Gamma\left(x, a_{-i}\right)$, so that in particular candidate $i$ is elected. $\triangleleft$ 
STEP 3. For no candidate $i$ does there exist a value of $x$ satisfying (1), (2), $\#\{j \in P$ : $\left.a_{j}=x\right\} \geq 2$, and

$$
\#\left\{j \in P: a_{j}=x\right\} \geq \#\left\{j \in P: a_{j}=a_{i}\right\} .
$$

Proof. If, on the contrary, some value of $x$ satisfies these conditions and candidate $i$ moves to $x$, then by Step 2 she remains elected and by (1) the policy outcome remains the same. Further, by (3) her payoff increases (given economies of party size), contradicting the fact that $(a, B)$ is a subgame persistent equilibrium.

STEP 4. The configurations of the candidates' positions consistent with subgame persistent equilibrium satisfy the conditions given in the proposition.

Proof. Reindex the candidates from $-k$ to $k$ in such a way that $a_{i} \leq a_{j}$ if $i \leq j$, omitting the index 0 if the number of candidates is even.

(i) If the number of candidates is even, the positions of candidates -1 and 1 differ: If the positions are the same, then the policy outcome remains the same if candidate 1 or candidate -1 withdraws, so the configuration is not an equilibrium by Lemma 3 .

(ii) If the number of candidates is odd, candidate 0 is an independent: If the positions of candidates 0 and 1 are the same, then the policy outcome remains the same if candidate -1 withdraws, so the configuration is not an equilibrium by Lemma 3 . Similarly, the positions of candidates -1 and 0 differ.

(iii) If for some $j \geq 1$ the number of candidates with position $a_{j}$ is at least 2 , then $a_{i}=a_{k}$ if $i \geq 2$ and $k \geq 2$ : Suppose to the contrary that $a_{i} \neq a_{k}$ and let $a_{k}$ be such that the number of candidates whose position is $a_{k}$ is at least the number whose position is $a_{i}$. If candidates $i$ and $k$ are both independents, let $x=a_{j}$; otherwise, let $x=a_{k}$. We claim that $x$ satisfies the conditions in Step 3, so that the configuration is not an equilibrium. Condition (1) is satisfied because $a_{i}$ and $x$ are on the same side of the median. To verify condition (2), note that if the action of a candidate is changed 
to nonparticipation, then the change in the median is the same whether candidate $i$ 's position is $a_{i}$ or $x$; in both cases, the median does not move outside the interval from the position of candidate -1 to the position of candidate 1 . Condition (3) is satisfied by the construction of $x$.

A symmetric argument shows if for some $j \leq-1$ the number of candidates with position $a_{j}$ is at least 2 , then $a_{i}=a_{k}$ if $i \leq-2$ and $k \leq-2$.

By (iii), all candidates $j$ with $j \geq 2$ are either independents or members of a single party, and similarly for all candidates $j$ with $j \leq-2$.

- Suppose these two sets are both parties. If there are two remaining candidates, -1 and 1 , then their positions are distinct by (i). Thus either these candidates take distinct positions as independents, or each belongs to one of the parties, or one is an independent and the other belongs to a party. If there are three remaining candidates, $-1,0$, and 1 , then their positions are distinct by (ii). Thus either these candidates take distinct positions as independents, or one of them belongs to one of the parties and the other two are independents, or one of them belongs to one party, another belongs to the other party, and the third is an independent.

- Suppose one of these two sets is a party whereas the members of the other set are independents. By (i) and (ii), at most one of the remaining candidates is a member of the party and the others are independents, so that the number of independents is at least as large as the number of members of the party.

- Suppose that both of the sets consist of independents. By (i) and (ii) the remaining candidates are independents, so all candidates are independents.

The proof is now complete.

Proof of Proposition 2. Let $(a, B)$ be a subgame IC-persistent equilibrium with positive voter turnout. As in the proof of Proposition 1, we start by showing that every candidate is elected. 
STEP 1. In $(a, B)$, every candidate is elected.

Proof. Suppose that in the equilibrium $(a, B)$, candidate $i$ is not elected. Then by Lemma 2, $B(a)$ is an equilibrium of the subgame $\Gamma\left(\theta, a_{-i}\right)$ following $\left(\theta, a_{-i}\right)$. Further, $\left(\left(\theta, a_{-i}\right), B(a)\right)$ is incentive compatible because $(a, B(a))$ is incentive compatible and the identities and positions of elected candidates are the same in the outcomes of these two strategy profiles. Therefore by subgame IC-persistence, $B\left(\theta, a_{-i}\right)=B(a)$, so that $i$ 's withdrawal is profitable, contradicting the fact that $(a, B)$ is an equilibrium. Thus every candidate is elected.

We now prove an analogue of Step 2 of the proof of Proposition 1. That is, we give conditions on $x$ such that when candidate $i$ moves to $x$, the citizens' voting behavior remains an equilibrium and the elected candidates' positions remain incentive compatible. In addition to the conditions in Step 2 of the proof of Proposition 1 we need the following condition.

For any $j \neq i$ with $a_{j} \in X$ and any $y \in X \cup\{\theta\}$ there exists $z \in X \cup\{\theta\}$ such that $M\left(z, a_{-j}\right)=M\left(y,\left(x, a_{-i}\right)_{-j}\right)$.

This condition says that any legislative outcome that can be induced by some candidate $j$ when candidate $i$ 's position is $x$ can also be induced by candidate $j$ when candidate $i$ 's position is $a_{i}$ (given that the position of every other candidate $k$ is $a_{k}$ in both cases). If the condition is satisfied and the citizens' voting profile $B$ is such that all candidates are elected both when their actions are $a$ and when they are $\left(x, a_{-i}\right)$, then if $(a, B(a))$ is incentive compatible, so is $\left(\left(x, a_{-i}\right)\right.$, $\left.B\left(x, a_{-i}\right)\right)$.

STEP 2. If $x$ satisfies (1), (2), and (4) for some candidate $i$ and $\left\{j \in P: a_{j}=x\right\} \geq 2$, then $B(a)$, the voting equilibrium of the subgame $\Gamma(a)$ following $a$ in the equilibrium $(a, B)$, is also an equilibrium of the subgame $\Gamma\left(x, a_{-i}\right)$ following $\left(x, a_{-i}\right)$, $M(A(a, B(a)))=M\left(A\left(\left(x, a_{-i}\right), B(a)\right)\right)$, and $\left(\left(x, a_{-i}\right), B(a)\right)$ is incentive compatible, so that $B\left(x, a_{-i}\right)=B(a)$ and in particular $i$ remains elected in $\Gamma\left(x, a_{-i}\right)$.

PROOF. By Step 2 of the proof of Proposition $1, B(a)$ is an equilibrium of $\Gamma\left(x, a_{-i}\right)$. The fact that $\left(\left(x, a_{-i}\right), B(a)\right)$ is incentive compatible follows from (4) and the fact 
that $(a, B(a))$ is incentive compatible (because $(a, B)$ is a subgame IC-persistent equilibrium). From the definition of subgame IC-persistence, we conclude that $B\left(x, a_{-i}\right)=B(a)$. By Step 1 , all candidates are elected in $\Gamma\left(x, a_{-i}\right)$, so that in particular candidate $i$ is elected.

The argument for the following step is the same as the argument for Step 3 of the proof of Proposition 1.

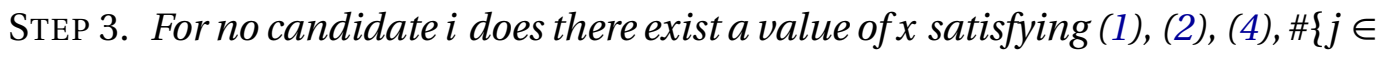
$\left.P: a_{j}=x\right\} \geq 2$, and (3).

STEP 4. The only configurations of the candidates' positions consistent with subgame IC-persistent equilibrium are those given in the proposition.

Proof. Reindex the candidates from $-k$ to $k$, as in the proof of Step 4 of the proof of Proposition 1. Points (i) and (ii) of that proof, which depend only on Lemma 3, remain correct. Point (iii) is replaced by the following two points.

(iii') If the number of candidates is odd and for some $j \geq 1$ the number of candidates with position $a_{j}$ is at least 2 , then $a_{i}=a_{k}$ if $i \geq 2$ and $k \geq 2$ : The position $x$ specified in the argument for (iii) in the proof of Step 4 of the proof of Proposition 1 satisfies the conditions in Step 2, except possibly (4), by the argument in that proof. To show that it satisfies (4), observe that any change in a candidate's action either does not affect the outcome or changes it from $a_{0}$ to some other point between the positions of candidates -1 and 1 , and the impact on the outcome is the same whether candidate $i$ 's position is $a_{i}$ or $x$.

A symmetric argument shows if the number of candidates is odd and for some $j \leq-1$ the number of candidates with position $a_{j}$ is at least 2 , then $a_{i}=a_{k}$ if $i \leq-2$ and $k \leq-2$.

(iii") If the number of candidates is even and for some $j \geq 1$ the number of candidates with position $a_{j}$ is at least 2 , then $a_{i}=a_{k}$ if $i \geq 3$ and $k \geq 3$ : Again we need to show only that the position $x$ specified in the argument for (iii) in the proof of Step 4 of the proof of Proposition 1 satisfies (4). 
38 Osborne and Tourky

Under the stated conditions, the positions to which a change in a candidate's action can change the outcome lie between the positions of candidates -2 and 2 whether candidate $i$ 's position is $a_{i}$ or $x$, and the positions of candidates $-2,-1,1$, and 2 are not affected by the change in candidate $i$ 's position (given $i \geq 3$ ). Further, as in the previous case the impact of any given change in a candidate's action is the same whether candidate $i$ 's position is $a_{i}$ or $x$. Thus the position $x$ satisfies (4).

A symmetric argument shows if the number of candidates is even, and for some $j \leq-1$ the number of candidates with position $a_{j}$ is at least 2 , then $a_{i}=a_{k}$ if $i \leq-3$ and $k \leq-3$.

If the number of candidates is odd, (iii') is the same as (iii) in Step 4 of the proof of Proposition 1, so that the set of configurations of the candidates' positions consistent with subgame IC-persistent equilibrium is the same as the set consistent with subgame persistent equilibrium: either (a) there are two parties, between which there are up to three independents, or (b) there is one party and at least as many independents as members of the party, and all independents are on the same side of the party, or (c) all candidates are independents.

If the number of candidates is even, (iii") differs from (iii) in that (iii") gives a condition under which all candidates $j$ with $j \geq 3$, rather than $j \geq 2$, belong to a single party and all candidates $j$ with $j \leq-3$, rather than $j \leq-2$, belong to a single party. Four candidates remain: $-2,-1,1$, and 2 . By point (i) of the proof of Step 4 of the proof of Proposition 1, the positions of candidates -1 and 1 differ. Thus the possible equilibrium configurations of positions are given as follows.

- If all candidates $j$ with $j \geq 3$ belong to a single party and all candidates $j$ with $j \leq-3$ belong to a single party, then either candidates 1 and 2 are both independents, or one of them belongs to the party on the right and the other is an independent, or both belong to the party on the right, or the two of them constitute a small party, and symmetrically for candidates -2 and -1 .

- If all candidates $j$ with $j \geq 3$ are independents whereas all candidates $j$ with $j \leq-3$ belong to a single party, then candidate 2 is an independent 
(otherwise all candidates $j$ with $j \geq 3$ belong to a single party by (iii")), and thus candidate 1 is also an independent (because her position differs from that of candidate -1 ). Candidates -1 and -2 are either both independents, or one belongs to the party on the left and the other is an independent, or both belong to the party on the left, or the two of them constitute a small party.

Symmetric considerations apply if all candidates $j$ with $j \leq-3$ are independents whereas all candidates $j$ with $j \geq 3$ belong to a single party.

- If all candidates $j$ with $j \geq 3$ or $j \leq-3$ are independents, then all candidates are independents: candidates -2 and 2 are independents by (iii") and candidates -1 and 1 are independents because their positions must differ.

The proof is now complete.

\section{REFERENCES}

Baron, David P. (1993), “Government formation and endogenous parties.” American Political Science Review, 87, 34-47. [10]

Cox, Gary W. (1987), “Duverger's law and strategic voting.” Unpublished paper, Department of Government, University of Texas, Austin. [8]

Duverger, Maurice (1954), Political Parties. Wiley, New York. [7]

Feddersen, Timothy J. (1992), "A voting model implying Duverger's law and positive turnout.” American Journal of Political Science, 36, 938-962. [8]

Jackson, Matthew O. and Boaz Moselle (2002), "Coalition and party formation in a legislative voting game." Journal of Economic Theory, 103, 49-87. [10]

Levy, Gilat (2004), “A model of political parties.” Journal of Economic Theory, 115, 250-277. [10]

Miller, Nicholas R. (1995), Committees, agendas, and voting. Harwood Academic, Chur, Switzerland. [3] 
40 Osborne and Tourky

Morelli, Massimo (2004), "Party formation and policy outcomes under different electoral systems.” Review of Economic Studies, 71, 829-853. [8]

Osborne, Martin J., Jeffrey S. Rosenthal, and Matthew A. Turner (2000), "Meetings with costly participation." American Economic Review, 90, 927-943. [10]

Palfrey, Thomas R. (1989), “A mathematical proof of Duverger's law.” In Models of strategic choice in politics (Peter C. Ordeshook, ed.), 69-91, University of Michigan Press, Ann Arbor. [8]

Snyder, Jr., James M. and Michael M. Ting (2002), "An informational rationale for political parties.” American Journal of Political Science, 46, 90-110. [10] 